\title{
Taxa real de câmbio, desalinhamento cambial e crescimento econômico no Brasil (1994-2007)
}

\author{
JOSÉ LUIS OREIRO \\ LIONELLO PUNZO \\ ELIANE ARAÚJO \\ GABRIEL SQUEFF*
}

Real exchange rate, exchange rate misalignment and economic growth in Brazil: 1994-2007. In this article we argue that the Brazilian economy presented in the period 1994-2007 a tendency of real exchange rate appreciation with respect to its equilibrium value, mainly from 2005 . This exchange rate misalignment has worked to reduce the growth of Brazilian economy and is the root of the re-emergence of current account deficits.

Keywords: exchange rate misalignment; economic growth; the Brazilian economy.

JEL Classification: F14; F31; F43.

\section{INTRODUÇÃO}

Neste artigo apresentaremos evidências empíricas a respeito da existência de um significativo desalinhamento cambial na economia brasileira, notadamente a partir de 2005. Ao contrário do que foi afirmado por alguns economistas de corte teórico mais ortodoxo (cf. Pastore et al., 2008), a apreciação experimentada pela taxa real de câmbio nos últimos anos não se deve apenas a uma apreciação do

\footnotetext{
* Respectivamente: professor do Departamento de Economia da Universidade de Brasília, pesquisador Nível I do CNPq e diretor da Associação Keynesiana Brasileira. E-mail: joreiro@unb.br. Professor da Universidade de Siena, Itália. E-mail: punzo@unisi.it. Professora do Departamento de Economia da Universidade Estadual de Maringá. E-mail: eliane.araujo@gmail.com. Técnico de Planejamento e Pesquisa do IPEA. E-mail: gabriel.squeff@ipea.gov.br. Os autores agradecem aos comentários de Márcio Holland (FGV-SP) a uma versão anterior deste artigo. Eventuais falhas são, contudo, de nossa inteira responsabilidade. Submetido: 20/5/2008: Aprovado 18/6/2010.
} 
valor de equilíbrio da taxa de câmbio, mas fundamentalmente a ocorrência de uma sobreapreciação do câmbio com respeito a taxa de câmbio de equilíbrio.

O desalinhamento cambial gera dois tipos de problemas para a economia brasileira. Em primeiro lugar, a sobreapreciação da taxa de câmbio é a razão fundamental para o reaparecimento do déficit em conta-corrente, a partir de 2007, trazendo de volta o fantasma da restrição externa ao crescimento. Em segundo lugar, a teoria econômica e a experiência internacional mostram que um desalinhamento cambial negativo, ou seja, uma situação na qual a taxa real de câmbio está abaixo de seu valor de equilíbrio, afeta negativamente o crescimento econômico no longo prazo. Isso porque a sobreapreciação cambial pode induzir um aumento do grau de concentração da estrutura produtiva em setores com baixo valor agregado e/ou baixo nível de conteúdo tecnológico. Se isso ocorrer, haverá uma redução da elasticidade-renda das exportações, o que levará a uma redução na taxa de crescimento de longo prazo da economia.

O presente artigo está estruturado em quatro seções, incluindo a presente introdução.

A segunda seção está dedicada à análise do comportamento da taxa real de câmbio no Brasil no período 1994-2007. Verificaremos que nos últimos 14 anos a taxa real de câmbio apresentou um desalinhamento crônico com respeito ao seu valor de equilíbrio, alternando períodos de sobreapreciação (1994-1998; 2005 2007) com períodos de relativo equilíbrio cambial (1999-2004).

A terceira seção está dedicada à análise dos efeitos de longo prazo do desalinhamento cambial. Mais especificamente iremos argumentar que a sobreapreciação cambial não só está por trás do reaparecimento dos déficits em conta-corrente, como, além disso, tem um claro efeito negativo sobre o crescimento da economia brasileira. Adicionalmente, a sobreapreciação pode estar induzindo um processo de desindustrialização, haja vista um aumento do grau de concentração da estrutura produtiva em setores com baixo valor adicionado e/ou baixo conteúdo tecnológico.

$\mathrm{Na}$ quarta seção fazemos uma revisão das conclusões obtidas no presente artigo.

\section{TAXA DE CÂMBIO DE EQUILÍBRIO E DESALINHAMENTO CAMBIAL NO BRASIL}

O desalinhamento cambial pode ser definido como uma situação na qual ocorrem desvios duradouros da taxa de câmbio real com relação à taxa de equilíbrio de longo prazo $^{1}$. Se a taxa de câmbio real é menor que o nível de equilíbrio, carac-

\footnotetext{
${ }^{1} \mathrm{O}$ conceito de taxa de câmbio de equilíbrio de longo-prazo é devido a Nurkse (1945). Na formulação deste autor, a taxa de câmbio de equilíbrio de longo-prazo corresponderia ao valor da taxa real de câmbio que garante o atendimento simultâneo de equilíbrio externo - situação na qual o déficit em conta-corrente pode ser financiado com um fluxo sustentável de entrada de capitais - e de equilíbrio interno, situação na qual a economia opera em condições de pleno emprego da força de trabalho. Em função das dificuldades existentes na estimação do pleno emprego da força de trabalho e do nível
} 
teriza-se uma situação de sobreapreciação cambial; se o contrário ocorre, tem-se a sub-apreciação cambial.

Apesar de o conceito de desalinhamento cambial ser aparentemente simples, sua estimação permanece como um dos principais desafios empíricos da macroeconomia aberta (Edwards, 1989; Hinkle e Montiel, 1999). A grande dificuldade reside no fato de que a taxa de câmbio de equilíbrio de longo prazo não é uma variável diretamente observável.

São três as principais medidas utilizadas na literatura econômica para estimar a taxa de câmbio de equilíbrio de longo prazo: (i) a abordagem da paridade do poder de compra (PPC); (ii) a abordagem de equilíbrio da conta-corrente; e (iii) a abordagem baseada nos fundamentos econômicos.

A medida de desalinhamento cambial baseada na PPC baseia-se no conceito de que na ausência de restrições ao comércio e de custos de transporte, os preços dos bens de uma economia, cotados em moeda estrangeira, não devem diferir dos preços (dos mesmos bens) praticados no exterior. Em que pese ser simples e de fácil mensuração, sérias desvantagens pesam contra esta abordagem, notadamente o fato de que se desconsideram os choques advindos de fatores reais que provoquem alterações nos preços relativos.

Já a abordagem do equilíbrio em conta-corrente taxa de câmbio de equilíbrio é aquela que pode tornar o saldo em conta-corrente equivalente ao fluxo líquido de capitais a médio e a longo prazos, considerando-se a antecipação das políticas macroeconômicas, os efeitos defasados de mudanças passadas na taxa de câmbio e a expectativa sobre outras variáveis. Suas principal desvantagem reside na dificuldade inerente à escolha da meta de equilíbrio para a conta-corrente. (Frankel e Goldstein, 1986).

Empregaremos neste artigo, deste modo, a abordagem calcada nos fundamentos econômicos. Esta abordagem se baseia na ideia de que sendo o equilíbrio de longo prazo uma situação em que as variáveis endógenas assumem valores que são sustentáveis ao longo do tempo, então os valores assumidos por essas variáveis deve depender apenas dos "fundamentos", ou seja, daquelas variáveis cuja influência sobre o valor das variáveis endógenas seja independente das expectativas dos agentes econômicos (Montiel, 1999, p. 221). Em outras palavras, o equilíbrio de longo prazo pressupõe a inexistência de bolhas especulativas, mesmo que tais bolhas sejam racionais ${ }^{2}$.

Essa metodologia foi desenvolvida por autores como Edwards (1989) e Baffes,

sustentável de déficit em conta-corrente, mais recentemente o conceito de taxa real de câmbio de equilíbrio de longo prazo passou a ser entendido como o nível da taxa real de câmbio determinado pelos "fundamentos econômicos". Em outras palavras, trata-se do nível da taxa real de câmbio para o qual as expectativas a respeito de valor futuro não têm influência na determinação da variável em consideração (Montiel, 1999, p. 223).

${ }^{2}$ Adicionalmente, o conceito de equilíbrio de longo prazo exige que os "fundamentos" se encontrem nos seus valores sustentáveis a longo prazo, ou seja, que se desconsidere os termos transitórios das variáveis de "fundamento". 
Elbadawi e O'Connel (1999), envolvendo, em geral, três etapas principais. $\mathrm{Na}$ primeira etapa, investiga-se a relação de longo prazo a ser estimada, adaptando a teoria existente às características da economia. Essa relação é representada por um modelo cujos parâmetros de longo prazo são estimados em uma segunda etapa, mediante técnicas apropriadas para as características das séries temporais utilizadas. $\mathrm{Na}$ terceira etapa, os parâmetros estimados são empregados para calcular a taxa de câmbio de equilíbrio de longo prazo.

A relação entre a taxa de câmbio real e os fundamentos econômicos pode ser expressa da seguinte forma:

$$
R E R_{t}=\alpha+\beta F U N D_{i t}+u_{t}
$$

Onde: RER é a taxa de câmbio real; $\alpha$ é o termo constante; $F U N D_{i t}$ é um vetor de variáveis fundamentais; e $u_{t}$ é o termo de erro.

Estimados os coeficientes referentes a cada fundamento, estes são utilizados para encontrar a taxa de câmbio real determinada pelos fundamentos macroeconômicos. A grande vantagem desse método é permitir que a taxa de câmbio de equilíbrio sofra mudanças ao longo do tempo conforme os fundamentos são alterados (Baffes, Elbadawi e O'Connel, 1999), contrariamente ao que ocorria na abordagem da PPC, na qual esta taxa era constante.

Deste modo, iremos agora proceder a determinação da taxa real de câmbio de equilíbrio de longo prazo para a economia brasileira.

Os dados utilizados nas estimações foram as séries de tempo trimestrais das seguintes variáveis: taxa de câmbio real efetiva (RER), deflacionada pelo INPC e calculada pelo IPEA; grau de abertura (OPEN), obtido pela soma das exportações com importações como proporção do PIB, calculado pelo IPEA; preço externo (PFOR) é definido como a variação dos preços externos em moeda nacional, construído a partir dos dados do Banco Central do Brasil e da Revista Conjuntura Econômica ${ }^{3}$; relação saldo da balança comercial/PIB (BCPIB), coletado junto ao IPEA; consumo do governo/PIB (CG), coletado pelo IBGE; termos de troca (TOT), obtido no IPEA; e diferencial de juros interno e externo (DIFJUR), obtido no World Economic Statistics. O período de análise considerado foi do quarto trimestre de 1994 ao terceiro trimestre de 2007.

$\mathrm{Na}$ equação estimada, a taxa de câmbio real é função de um conjunto de variáveis exógenas e de políticas, implicando que a taxa de câmbio real esperada resultante pode variar ao longo do período, seguindo mudanças nos fundamentos econômicos, conforme equação (2).

$$
R E R=\beta_{0}+\beta_{1} T O T+\beta_{2} O P E N+\beta_{3} D I F J U R+\beta_{4} C G+\beta_{5} P F O R+\beta_{6} B C P I B
$$

\footnotetext{
${ }^{3}$ A série PFOR foi calculada multiplicando o Índice de Preços por Atacado - Oferta Global (IPAOG), índice (ago. $1994=100$ ) - FGV/Conj. Econômica (2008) - pela taxa de câmbio R \$ US\$ - BCB Boletim/BP (2008).
} 
Os resultados esperados da influência de cada variável na taxa de câmbio real são os seguintes:

$$
\frac{\partial R E R}{\partial T O T}<0 ; \frac{\partial R E R}{\partial O P E N}>0 ; \frac{\partial R E R}{\partial P F O R}<0 ; \frac{\partial R E R}{\partial D I F J U R}<0 ; \frac{\partial R E R}{\partial B C P I B}<0 ; \frac{\partial R E R}{\partial C G}<0
$$

Segundo a literatura empírica, uma melhora nos temos de troca (TOT) aprecia a taxa de câmbio real, sendo que o efeito gasto desta variável domina o efeito substituição (Edwards, 1989).

A influência esperada de $B C P I B$ sobre a taxa de câmbio é negativa, porque, quanto maior o saldo da balança comercial, maior será a entrada de divisas e, em consequência, menor o câmbio.

OPEN mede o grau em que o país é afetado pelo ambiente internacional. Em geral, uma depreciação da taxa de câmbio ocorre quando o nível de abertura é alto. A razão para isso é que uma liberalização comercial reduz os preços domésticos dos bens comercializáveis com o exterior, alterando sua demanda. Em equilíbrio, o preço dos bens deve cair e o câmbio se depreciar.

Já PFOR mede a variação do preço externo em moeda nacional. Um aumento dessa variável aumenta os preços domésticos dos bens comercializáveis externamente, causando mudança na demanda em direção oposta a esses bens. Assim, um aumento em PFOR tende a apreciar o câmbio.

Uma mudança permanente no tamanho do governo $(C G)$ afeta o câmbio real quando altera a demanda por bens comercializáveis e não comercializáveis. Nos países onde um maior gasto do governo aumenta a demanda por bens não comercializáveis, a taxa de câmbio se aprecia.

Por fim, quando a taxa de juros de um país está acima da taxa de juros internacional (DIFJUR), há um maior retorno para os capitais externos dentro do país, o que provoca a entrada de capitais na economia e a consequente apreciação do câmbio.

O teste de raiz unitária Augmented Dickey Fuller (ADF) (Tabela 1) indicou que todas as séries são integradas de ordem um ${ }^{4}$.

Tabela 1: Teste de Raiz Unitária (ADF) - nível e diferença

\begin{tabular}{lccccc}
\hline \multirow{2}{*}{ Variável } & \multirow{2}{*}{ Estatística $(\tau)$} & P-Valor & \multicolumn{3}{c}{ Valores Críticos } \\
\cline { 4 - 6 } & $-1,4699$ & 0,5405 & $-3,5683$ & $-2,9212$ & $-2,5986$ \\
RER & $-2,1458$ & 0,2285 & $-3,5925$ & $-2,9314$ & $-2,6039$ \\
OPEN & $-1,1299$ & 0,6951 & $-3,5966$ & $-2,9332$ & $-2,6049$ \\
DIFJUR & $-1,3315$ & 0,6073 & $-3,5777$ & $-2,9252$ & $-2,6007$ \\
BCPIB & $-1,8695$ & 0,3437 & $-3,5655$ & $-2,9200$ & $-2,5979$ \\
TOT & $-0,9823$ & 0,7527 & $-3,5683$ & $-2,9212$ & $-2,5986$ \\
PFOR & $-0,1131$ & 0,9418 & $-3,5777$ & $-2,9252$ & $-2,6007$ \\
CG & & & & & \\
\hline
\end{tabular}

\footnotetext{
${ }^{4}$ Todos os testes econométricos deste artigo foram feitos com o software Eviews na versão 6.0. Para maiores informações a respeito da metodologia empregada na pesquisa ver Enders (1995); Stock e Watson (2004).
} 


\begin{tabular}{lccccc}
\hline $\begin{array}{c}\text { Primeira diferença da } \\
\text { variável }\end{array}$ & \multirow{2}{*}{ Estatística $(\tau)$} & P-Valor & \multicolumn{3}{c}{ Valores Críticos } \\
\cline { 4 - 6 } & $-5,3819$ & 0,0000 & $-3,5683$ & $-2,9212$ & $-2,5986$ \\
DRER & $-8,2680$ & 0,0000 & $-3,5925$ & $-2,9314$ & $-2,6039$ \\
DOPEN & $-4,4010$ & 0,0011 & $-3,5966$ & $-2,9332$ & $-2,6049$ \\
DDIFJUR & $-2,6138$ & 0,0801 & $-3,5777$ & $-2,9252$ & $-2,6007$ \\
DBCPIB & $-6,3965$ & 0,0000 & $-3,5655$ & $-2,9200$ & $-2,5979$ \\
DTOT & $-6,0607$ & 0,0000 & $-3,5683$ & $-2,9212$ & $-2,5986$ \\
DPFOR & $-2,6809$ & 0,0927 & $-3,5777$ & $-2,9252$ & $-2,6007$ \\
DCG &
\end{tabular}

Fonte: Elaboração própria com base nos dados da pesquisa.

Deste modo, foram realizados os testes de cointegração que indicaram que as variáveis não estacionárias do modelo estão apresentando trajetórias comuns ou em bloco, de forma que no longo prazo há uma relação estável entre essas variáveis (Tabela 2).

Tabela 2: Teste de Johansen

\begin{tabular}{ccccc}
\hline & \multicolumn{2}{c}{ Máximo Valor } & \multicolumn{2}{c}{ Estatística Traço } \\
\cline { 2 - 5 } & Valor Observado & Valor Crítico 5\% & Valor Observado & Valor Crítico 5\% \\
\hline$r=0$ & 81.33586 & 50.59985 & 209.5281 & 150.5585 \\
$R \leq 1$ & 44.03660 & 44.49720 & 128.1923 & 117.7082 \\
$R \leq 2$ & 27.51252 & 38.33101 & 84.15567 & 88.80380 \\
\hline
\end{tabular}

Fonte: Elaboração própria com base nos dados da pesquisa.

Logo, podemos trabalhar com as séries em nível usando o método de Mínimos Quadrados Ordinários (MQO) para estimar os parâmetros da regressão ${ }^{5}$. As estimativa da equação (2) está reportada na Tabela 3.

Tabela 3: Método de Mínimos Quadrados Ordinários

\begin{tabular}{lccccccc}
\hline Estatísticas & \multicolumn{7}{l}{ Variáveis } \\
\cline { 2 - 8 } & C & OPEN & DIFJUR & BCPIB & TOT & PFOR & CG \\
\hline & 202,84 & 1,754 & $-0,219$ & $-4,795$ & $-1,433$ & -0.070 & $-0,0003$ \\
\hline Erro-padrão & 26,661 & 0,510 & 0,112 & 0,978 & 0,229 & 0.009 & 0,0001 \\
Estatística t & 7,608 & 3,441 & $-1,95$ & $-4,902$ & $-6,264$ & 7.927 & $-4,050$ \\
P-valor & 0,000 & 0,001 & 0,058 & 0,000 & 0,000 & 0.000 & 0,0002 \\
\hline
\end{tabular}

continua na pág. 558

\footnotetext{
${ }^{5}$ Segundo Hamilton (1994), se as séries do modelo possuem essas características, o método de MQO continua sendo um estimador superconsistente. Para uma demonstração formal a esse respeito, ver Hamilton (1994, p. 587).
} 


\begin{tabular}{lc}
\hline R2 & 0,9311 \\
R2 ajustado & 0,9219 \\
Teste F & 101,346 \\
Probabilidade & $(0,000)$ \\
Durbin Watson & 1,8224 \\
\hline
\end{tabular}

Fonte: Dados da pesquisa.

Com relação aos resultados, verifica-se que a hipótese de nulidade individual pode ser rejeitada para todos os coeficientes com nível inferior a 5\% de significância. Quanto ao valor esperado dos parâmetros, observou-se que as estimativas dos coeficientes apresentaram os sinais previstos em (3).

O Gráfico 1 mostra a evolução da taxa real efetiva de câmbio e da taxa de câmbio de equilíbrio entre o quarto trimestre de 1994 e o quarto trimestre de 2007.

Gráfico 1: Taxas de câmbio efetiva real e esperada real (índice - média de $2000=100$ )



Fonte: Dados da pesquisa.

No Gráfico 1, observa-se que houve uma significativa sobreapreciação cambial no Brasil nos períodos (1994:4 - 1998:4) e (2005:1 - 2007:4). Além disso, verificamos que no período 1994-1999 a taxa real de câmbio de equilíbrio mostrou uma tendência nítida a depreciação, a qual foi ligeiramente revertida no período 20052007. Daqui se segue que é incorreta a afirmação de Pastore et al. (2008, p. 283) de que os movimentos da taxa real de câmbio efetiva no Brasil seriam, na sua essência, decorrentes da apreciação da taxa real de câmbio de equilíbrio. Embora a mesma tenha se valorizado nos últimos três anos, a apreciação da taxa real de câmbio efetiva superou em muito a apreciação da taxa real de câmbio de equilíbrio, dando origem a um desalinhamento cambial significativo, estimado em cerca de $18 \%$ no quarto trimestre de 2007. 


\section{EFEITOS DO DESALINHAMENTO CAMBIAL}

Nesta seção investigaremos os efeitos do desalinhamento cambial sobre a economia brasileira, particularmente sobre a taxa de crescimento econômico, o saldo em conta-corrente e a estrutura produtiva.

Inicialmente, analisaremos o impacto do desalinhamento cambial sobre o crescimento do produto real. Para tanto, a taxa de crescimento da economia brasileira (GPIB) será regredida contra um conjunto de variáveis, entre elas o desalinhamento cambial. A regressão estimada será a seguinte:

$$
\mathrm{GPIB}=\alpha+\beta_{1} \mathrm{INV}+\beta_{2} \mathrm{EXPORT}+\beta_{3} \mathrm{CGOV}+\beta_{4} \mathrm{DES}+\mathrm{u}_{\mathrm{i}}
$$

Onde: GPIB é a taxa de crescimento do PIB; INV é a taxa de investimento (FBKF/PIB); EXPORT é a taxa de crescimento das exportações; CGOV é a taxa de crescimento do gasto de consumo do governo sobre o PIB e DES é a taxa de desalinhamento cambial, ou seja, o desalinhamento cambial dividido pelo câmbio de equilíbrio. Todas essas séries, exceto DES, foram coletadas no IPEA e são de periodicidade trimestral.

Para analisar a ordem de integração das séries novamente aplicou-se o teste $\mathrm{ADF}$, que indicou que as mesmas são estacionárias, conforme Tabela 4.

Tabela 4: Teste Dickey-Fuller Aumentado (ADF) - na primeira diferença

\begin{tabular}{ccccc}
\hline Variável & T Calculado & Valor Crítico (1\%) & Valor Crítico (5\%) & Valor Crítico (10\%) \\
\hline CGO & -4.42 & -3.57 & -2.95 & -2.60 \\
EXPOT & -4.03 & -3.57 & -2.95 & -2.60 \\
INV & -3.49 & -3.57 & -2.95 & -2.60 \\
PIB & -8.27 & -3.57 & -2.95 & -2.60 \\
DES & -2.64 & -3.57 & -2.95 & -2.60 \\
SOBREVAL & -2.85 & -3.57 & -2.95 & -2.60 \\
\hline
\end{tabular}

Fonte: Dados da pesquisa

Objetivando corrigir a possível existência de viés ou inconsistência na estimativa por MQO, a equação (4) é estimada também pelo método das Variáveis Instrumentais (VI). Os resultados de ambas metodologias estão apresentados na Tabela 5.

Observa-se que os parâmetros dos dois modelos apresentaram os sinais esperados pela teoria kaldoriana do crescimento puxado pela demanda agregada, bem

\footnotetext{
${ }^{6}$ Estamos considerando a "visão kaldoriana" sobre crescimento de longo prazo segundo a qual o crescimento de longo prazo é determinado pela taxa de expansão da demanda agregada autônoma. Numa economia aberta, a demanda autônoma é dada pelas exportações e pelos gastos de consumo do governo. O investimento em capital fixo faz parte da assim chamada "demanda induzida", cabendo a ele o papel de "sustentador" do crescimento de longo prazo. A esse respeito ver Kaldor (1988), Thirwall (2002) e Oreiro et al. (2007).
} 
como foram estatisticamente significativos ${ }^{7}$. Quanto aos efeitos da variável desalinhamento cambial sobre o crescimento econômico verifica-se uma relação negativa entre essas variáveis, isto é, quanto maior a taxa de desalinhamento cambial menor tende a ser o crescimento econômico.

O desalinhamento cambial verificado na economia brasileira pode ser entendido também como uma medida de sobreapreciação cambial, haja vista que é a diferença entre a taxa de câmbio observada e a taxa de câmbio ajustada pelos fundamentos, que é negativa ao longo de quase todo o período analisado. Diversas pesquisas têm encontrado que taxas de câmbio sobrevalorizadas estão associadas a menores taxas de crescimento econômico, citando-se como exemplo Frenkel (2004) e Razin e Collins (1997).

Sendo assim, é interessante testar como o crescimento econômico responde a sobreapreciação cambial. Para tanto, a equação (4) foi reestimada, substituindo-se a séries do desalinhamento cambial $(D E S)$ pela série de sobreapreciação cambial (SOBREVAL). Para criar esta variável utilizamos a série do desalinhamento cambial somente com os valores negativos da série DES (taxa de desalinhamento cambial). Os resultados desta estimativa encontram-se na Tabela 6 .

Tabela 5: Regressão de Crescimento I

\begin{tabular}{|c|c|c|}
\hline Variável & MOO & MVI \\
\hline INV & 0.197733 & 0.11711 \\
& {$[0.071899]$} & {$[0.05921]$} \\
& $(2.750579)^{* *}$ & $(1.977826)^{* *}$ \\
EXPORT & 0.266595 & 0.233294 \\
& {$[0.056591]$} & {$[0.055152]$} \\
& $(4.710873)^{*}$ & $(4.230035)^{*}$ \\
CGO & 0.010415 & 0.008911 \\
& {$[0.002655]$} & {$[0.002598]$} \\
& $(3.922720)^{*}$ & $(3.430203)^{* *}$ \\
DES & -0.116802 & -0.110111 \\
& {$[0.067390]$} & {$[0.068325]$} \\
& $(-1.73225)^{* *}$ & $(-1.62809)^{* * *}$ \\
PIB(-1) & 0.068593 & 0.069599 \\
& {$[0.036474]$} & {$[0.035365]$} \\
& $(1.880627)^{* * *}$ & $(1.96801)^{* *}$ \\
C & 0.344253 & 0.214945 \\
\hline
\end{tabular}

Tabela 6: Regressão de Crescimento II

\begin{tabular}{|c|c|c|}
\hline Variável & MOO & VI \\
\hline INV & 0.203453 & 0.211330 \\
& {$[0.067779]$} & {$[0.07198]$} \\
EXPORT & $(2.857886)^{* *}$ & $(2.936096)^{* *}$ \\
& 0.232383 & 0.346081 \\
& {$[0.052385]$} & {$[0.060676]$} \\
CGO & $(4.656563)^{*}$ & $(5.703727)^{*}$ \\
& 0.010367 & 0.011569 \\
SOBREVAL & {$[0.002612]$} & {$[0.002581]$} \\
& $3.877620^{*}$ & $(4.482158)^{*}$ \\
& -0.1213536 & -0.244340 \\
PIB(-1) & {$[0.073210]$} & {$[0.131764]$} \\
& $(-1.72335)^{* * *}$ & $(-1.854370)^{* * *}$ \\
& 0.0672253 & 0.088049 \\
C & {$[0.016643]$} & {$[0.036154]$} \\
& $(1.825465)^{* * *}$ & $(2.435355)^{* *}$ \\
& 0.331783 & 0.350143 \\
\hline
\end{tabular}

continua na pág. 560

\footnotetext{
${ }^{7}$ A variável taxa de crescimento do PIB defasada (PIB-1) foi introduzida como variável explicativa na equação (4) para solucionar o problema de autocorrelação do modelo.
} 


\begin{tabular}{|c|c|c|c|c|c|}
\hline & $\begin{array}{c}\text { [0.118065] } \\
(2.915798)^{* *}\end{array}$ & $\begin{array}{c}{[0.098481]} \\
(2.182608)^{* *}\end{array}$ & & $\begin{array}{c}{[0.108065]} \\
(2.861987)^{* *}\end{array}$ & $\begin{array}{c}{[0.118797]} \\
(2.947391)^{* *}\end{array}$ \\
\hline R2 & 0.393 & 0.3481 & $\mathrm{R} 2$ & 0.399 & 0.520 \\
\hline R2 Ajustado & 0.3292 & 0.293 & R2 Ajustado & 0.331 & 0.459 \\
\hline Estatística F & 6.104 & 6.184 & Estatística F & 6.159 & 8.481 \\
\hline Prob. (teste F) & 0.000196 & 0.000195 & Prob. (teste F) & 0.000196 & 0.000017 \\
\hline Durbin-Watson & 2.283 & 2.346 & Durbin-Watson & 2.283 & 2.385 \\
\hline
\end{tabular}

Fonte: Dados da pesquisa

Nota: ${ }^{*}$ significativo a $1 \%,{ }^{*}$ significativo a $5 \% e^{* * *}$ significativo a $10 \%$.

Erro-padrão entre colchetes e teste T entre parênteses.

Segundo as estimativas realizadas pelos métodos de MQO e VI verifica-se que o coeficiente da variável sobreapreciação cambial é negativo e estatisticamente significante em ambos os casos. Além disso, este coeficiente é mais elevado que o coeficiente de desalinhamento cambial, indicando que o crescimento econômico é mais prejudicado por desalinhamentos cambiais que implicam sobreapreciação do câmbio.

Além de afetar o crescimento econômico, o desalinhamento cambial pode estar contribuindo para que outros problemas surjam no cenário macroeconômico. $\mathrm{O}$ saldo em transações correntes é exemplo mais proeminente. A partir de meados de 2003, período no qual a taxa de câmbio estava subvalorizada, o saldo em transações correntes como proporção do PIB acumulado em 12 meses foi positivo pela primeira vez desde o final de 1994. Após atingir o pico de 1,93\% em abril de 2005 observa-se uma trajetória predominantemente declinante deste indicador, de modo que a partir de janeiro do presente ano temos déficit em transações correntes com tendência acelerada de deterioração.

Segundo Pastore et al. (2008), esse comportamento do déficit em conta-corrente não seria o resultado de uma apreciação da taxa de câmbio, mas a consequência natural da "escassez de poupança doméstica" para o financiamento do investimento produtivo. Essa escassez de poupança é que estaria por trás da apreciação da taxa de câmbio, a qual seria decorrente, portanto, de uma apreciação do câmbio de equilíbrio, ao invés de ser o reflexo de um suposto desalinhamento cambial.

Contudo, os testes econométricos apresentados na seção anterior mostram a existência de um desalinhamento cambial significativo no Brasil a partir do segundo trimestre de 2005. Dessa forma, o comportamento do saldo da conta-corrente do balanço de pagamentos não pode ser unicamente atribuído a "escassez de poupança doméstica”. O desalinhamento cambial desempenha um papel importante, talvez até preponderante, no comportamento da conta em transações correntes.

Deve-se observar que a redução do saldo em conta-corrente do balanço de pagamentos coincide temporalmente com a ocorrência de uma sobreapreciação da taxa real de câmbio. Além disso, devemos ressaltar também que tomando como base o comportamento da taxa de crescimento das exportações e das importações 
a partir de 2006, podemos constatar a existência de uma tendência de médio prazo a redução do saldo da balança comercial e, portanto, uma tendência à deterioração crescente do saldo em conta-corrente. Com efeito, desde 2006 que a taxa de crescimento das importações supera a taxa de crescimento das exportações, reduzindo paulatinamente o resultado da balança comercial. Comparando-se o primeiro semestre de 2008 com o mesmo período em 2007, observa-se um crescimento das exportações de $23,8 \%$ vis-à-vis uma taxa de variação das importações de $50,6 \%$, resultando numa redução do saldo da balança comercial de $44,8 \%$. Se essa tendência de redução do saldo da balança comercial persistir nos próximos anos, a economia brasileira estará de volta com o "velho problema" da restrição externa, o qual foi um dos responsáveis pelo baixo crescimento observado nos últimos 20 anos.

A sobreapreciação do câmbio também parece estar afetando a estrutura da pauta de exportações brasileiras haja vista a redução da participação dos setores de alta e média tecnologia no total de exportações, conforme Tabela 7.

Tabela 7: Balança Comercial da Indústria de Transformação em US\$ bilhões

\begin{tabular}{l|cccccc}
\hline & 2003 & 2004 & 2005 & 2006 & 2007 & 2008 \\
\hline Baixa & 19.856 & 25.197 & 28.727 & 31.927 & 34.761 & 39.559 \\
Média-baixa & 5.488 & 8.871 & 10.258 & 10.545 & 9.185 & 5.118 \\
Média-alta & -3.376 & -2.531 & 443 & -897 & -10.344 & -30.190 \\
Alta & -5.245 & -7.484 & -8.320 & -11.779 & -14.824 & -21.653 \\
Prods. Ind. transformação & 16.723 & 24.053 & 31.107 & 29.796 & 18.779 & -7.166 \\
\hline
\end{tabular}

Fonte: IEDI (2009)

$\mathrm{Na}$ medida em que os produtos de alta e média intensidade tecnológica possuem uma maior elasticidade-renda, segue-se que a redução da participação desses produtos na pauta de exportações da economia brasileira deverá conduzir a uma redução da elasticidade-renda das exportações brasileiras, contribuindo para agravar o problema do desequilíbrio entre a taxa de crescimento das exportações e das importações.

\section{CONCLUSÃO}

Neste artigo mostramos que a economia brasileira apresenta uma tendência a apreciação da taxa real de câmbio, notadamente a partir de 2005. Esse desalinhamento tem efeitos negativos sobre o crescimento do produto real e tem atuado no sentido de reduzir o saldo em conta-corrente, ameaçando assim o equilíbrio externo da economia brasileira. 


\section{REFERÊNCIAS BIBLIOGRÁFICAS}

ARTUS, J. R; KNIGHT, M. D. (1984). "Issues in the assessment of exchange rates of the industrial countries", Occasional. Paper n. 29. Washington: IMF.

BAFFES; ELBADAWI; O'CONNEL (1999). Chapter 11. In: MONTIEL,P; HINKLE,L (orgs.). Exchange Rate Misalignment: concepts and measurement for developing countries. A World Bank Research Publication, Oxford.

BANCO CENTRAL DO BRASIL. Economia e Finanças: séries temporais. 2008.

EDWARDS, S. (1989). Real Exchange Rates, Devaluation, and Adjustment: Exchange Rate Policy in Developing Countries. Cambridge, MA: MIT Press.

ENDERS, W. (1995). Applied Econometric Times Series. New York: John Wiley e Sons, Inc., $2^{\text {a }}$ Edição.

FMI - INTERNATIONAL MONETARY FUND. World Economic Outlook. Washington, 2007.

FRANKEL, J. e GOLDSTEIN, M. (1986). A Guide to Target Zones. IMF StaffPapers, 33.

FRENKEL, R., (2004) "Real exchange rate and employment in Argentina, Brazil, Chile and Mexico", Cedes, Buenos Aires, paper presented to the G24.

FUNCEX. FUNDAÇÃO CENTRO DE ESTUDOS DO COMÉRCIO EXTERIOR. Disponível em: $<$ http://www.funcex.com.br/>.

HAMILTON, J. D. Time series analysis. Princeton University Press, 1994.

HARROD, R. (1939). “An Essay in Dynamic Theory”. The Economic Journal, vol. 49.

HINKLE, L. E., e MONTIEL, P. J. (1999). Exchange Rate Misalignment: Concepts and Measurement for developing countries. A World Bank Research Publication, Oxford University Press.

IBGE. INSTITUTO BRASILEIRO DE GEOGRAFIA E ESTATÍSTICA, 2008.

IPEA. Indicadores IPEA. Disponível em <http://www.ipeadata.gov.br.htm>, 2008.

KALDOR, N. (1988). "The role of effective demand in the short and in the long-run" In: Barrére, A. (org.) The Foundations of Keynesian Analysis. Macmillan Press: Londres.

MONTIEL, P. (1999). "The Long-run equilibrium real exchange rate: conceptual issues and empirical research” In: HINKLE, L. E., e MONTIEL, P. J. Exchange Rate Misalignment: Concepts and Measurement for developing countries. A World Bank Research Publication, Oxford University Press.

NURKSE, R. (1945). "Conditions of international monetary equilibrium”. Essays in International Finance 4 (Spring). Princeton, Nova Jersey: Princeton University Press, International Finance Section.

OREIRO, J. L; NAKABASHI, L; SILVA; G. J; LEMOS, B. P. (2007). “A Macroeconomia do Crescimento Puxado pela Demanda Agregada: Teoria e Aplicações ao Caso Brasileiro”. Anais do XXXV Encontro Nacional de Economia, Recife.

PASTORE, A. C.; PINOTTI, M. C.; ALMEIDA, L. P. (2008). "Câmbio e crescimento: o que podemos aprender?” In: BARROS, O.; GIAMBIAGI, F. (orgs.). Brasil Globalizado. Campus: Rio de Janeiro.

RAZIN, O.; COLLINS, S. (1997). Real Exchange Rate Misalignment and Growth, forthcoming. In: RAZIN, A. e SADKA, E. (eds.). International Economic Integration: Public Economics Perspectives, Cambridge University Press, also at NBER Working Paper, n. 6147, 1997.

RODRIK, D. (2007). The Real Exchange Rate and Economic Growth: Theory and Evidence. Disponível em <http://ksghome.harvard.edu/ drodrik/RER\%20and\%20growth.pdf >. Acesso em: 20 de outubro de 2007.

Stock, J. H.; Watson, M. W. (2004). Econometria. Sao Paulo: Addison Wesley.

THIRWALL, A. (2002). The Nature of Economic Growth. Edward Elgar: Aldershot.

WOOLDRIDGE, J. (2000). Econometric Analysis of Cross Section and Panel Data. Cambridge, Mass.:

The MIT Press. 